\title{
Serum Syndecan-1 Reflects Organ Dysfunction in Critically III Patients
}

\section{Keiko Suzuki}

Gifu University Hospital

\section{Hideshi Okada}

Gifu University

\section{Kazuyuki Sumi}

Gifu University Hospital

Hiroyuki Tomita

Gifu University

Ryo Kobayashi

Gifu University Hospital

Takuma Ishihara

Gifu University Hospital

Yoshinori Kakino

Gifu University

Kodai Suzuki

Gifu University

Naomasa Yoshiyama

Gifu University

Ryu Yasuda

Gifu University

Yuichiro Kitagawa

Gifu University

\section{Tetsuya Fukuta}

Gifu University

Takahito Miyake

Gifu University

Haruka Okamoto

Gifu University

Tomoaki Doi

Gifu University

Takahiro Yoshida

Gifu University

Shozo Yoshida 
Gifu University

\section{Shinji Ogura}

Gifu University

\section{Akio Suzuki ( $\nabla$ akio@gifu-u.ac.jp )}

Department of Pharmacy, Gifu University Hospital, 1-1 Yanagido, Gifu 501-1194, Japan.

\section{Research Article}

Keywords: Syndecan-1 (SDC-1), endothelial glycocalyx, creatinine, blood urea nitrogen (BUN), alanine transaminase

Posted Date: December 18th, 2020

DOI: https://doi.org/10.21203/rs.3.rs-128024/v1

License: (c) (i) This work is licensed under a Creative Commons Attribution 4.0 International License. Read Full License

Version of Record: A version of this preprint was published at Scientific Reports on April 23rd, 2021. See the published version at https://doi.org/10.1038/s41598-021-88303-7. 


\section{Abstract}

Syndecan-1 (SDC-1) is found in the endothelial glycocalyx and shed into the blood during systemic inflammatory conditions. We examined organ dysfunction associated with changing serum SDC-1 levels in critically ill patients. A single-center, retrospective, observational study was conducted at Gifu University Hospital. Patients admitted to the intensive care unit from March 2019 to February 2020 were enrolled. To evaluate the effect of SDC-1 on laboratory parameters measured the day after SDC-1 measurement with consideration for repeated measures, linear mixed effects models were constructed with each parameter as an outcome variable. A total of 94 patients were enrolled, and 831 samples were obtained. Analysis using mixed effects models for repeated measures with adjustment for age and sex showed that serum SDC-1 levels measured the day before significantly affected several outcomes, including aspartate aminotransferase (AST), alanine transaminase (ALT), creatinine (CRE), blood urea nitrogen (BUN), antithrombin III, fibrin degradation products, and D-dimer. Moreover, serum SDC-1 levels from the day before significantly modified the effect between time and several outcomes, including AST, ALT, CRE, and BUN. Serum SDC-1 may be a useful biomarker for daily monitoring of critically ill patients with kidney, liver and coagulation system injuries.

\section{Introduction}

The vascular endothelium, which consists of a thin monolayer of endothelial cells, lines the entire circulatory system from the heart to the smallest capillaries where it is exposed to the circulating blood. All healthy endothelia are coated with the sugar protein glycocalyx, which is composed of membranebound proteoglycans, glycoproteins, glycosaminoglycans, and adherent plasma proteins. ${ }^{1-4}$ It performs several functions that are necessary for vascular homeostasis, including regulation of vascular permeability and microvascular tone, inhibition of microvascular thrombosis, and regulation of leukocyte adhesion and migration on the endothelium. ${ }^{5-8}$

Systemic inflammatory conditions lead to endothelial dysfunction, which in turn increases paracellular permeability and the outflow of albumin/fluid into the interstitial space. ${ }^{5}$ This effect is thought to be caused by glycocalyx disruption. Schmidt et al. demonstrated that degradation of endothelial glycocalyx contributes to the pathogenesis of acute respiratory distress syndrome in the lipopolysaccharide (LPS)induced experimental sepsis mouse model and humans. ${ }^{9,10}$ Similarly, recent reports indicate that severe disruption to capillary endothelial glycocalyx leads to damaged organs, including the heart, kidneys, lungs, liver, and brain, as observed by scanning and transmission electron microscopy, suggesting that endothelial dysfunction occurs in the process of organ damage. ${ }^{11-13}$ Therefore, glycocalyx fragments shed into the blood during sepsis may serve as a clinically relevant biomarker of organ dysfunction, given the pathophysiologic implications of glycocalyx degradation. ${ }^{14}$

Serum syndecan-1 (SDC-1), the core protein in heparan sulfate proteoglycan, is found in the endothelial glycocalyx. In clinical research, serum SDC-1 has been used as an endothelial injury marker of several diseases, including chronic kidney disease, ${ }^{15}$ diabetes, ${ }^{16,17}$ cardiovascular disease, ${ }^{18}$ 
hypertriglyceridemia ${ }^{19}$ and sepsis. ${ }^{20,21}$ Moreover, several indicators have demonstrated that serum SDC-1 levels are significantly correlated with the Sequential Organ Failure Assessment (SOFA) score in patients with sepsis, ${ }^{22-24}$ and that non-survivors have significantly higher SDC-1 levels than survivors with sepsis. ${ }^{21,24,25}$ However, it remains unknown which organs show changing serum SDC-1 levels when damaged, and whether SDC-1 is a useful predictive marker for early detection of dysfunction in these organs.

The aim of this study was to investigate the association between organ dysfunction and changing serum SDC-1 levels in critically ill patients and to identify organ dysfunctions that may be amenable to early detection through daily monitoring of SDC-1 levels.

\section{Results}

\section{Patient characteristics}

Patient characteristics are shown in Table 1. A total of 94 patients (70 men and 24 women) were enrolled in this study, and 831 samples were obtained. The median age was 67.0 years (IQR, 53.3-77.0), median SOFA score on ICU admission was 6.0 (IQR, 4.0-9.8), and median duration of ICU stay was 10.5 days (IQR, 6.0-19.0). The most common condition upon admission was trauma $(n=32,34.0 \%)$, followed by myocardial infarction $(n=15,16.0 \%)$, soft tissue infection $(n=7,7.4 \%)$, burn $(n=6,6.4)$, heatstroke $(n=5$, $5.3 \%)$, heart failure $(n=3,3.2 \%)$, loss of consciousness $(n=2,2.1 \%)$, acute aortic dissection $(n=2,2.1 \%)$ and ventricular fibrillation $(n=2,2.1 \%)$.

\section{Association of serum SDC-1 levels measured the day before with various parameters}

The results of the mixed effects models with adjustment for age and sex are shown in Table 2. Serum SDC-1 levels measured the day before laboratory parameter measurements were significantly affected AST [coefficient $(\beta)=0.0042,95 \%$ confidence interval $(\mathrm{Cl})=0.0018-0.0065, P=0.001$ ], ALT $(\beta=0.0034$, $95 \% \mathrm{Cl}=0.0012-0.0057, P=0.003), \mathrm{CRE}(\beta=0.002,95 \% \mathrm{Cl}=0.0012-0.0028, P<0.001), \mathrm{BUN}(\beta=0.0015$, $95 \% \mathrm{Cl}=0.0002-0.0028, P=0.019)$, T-Bil $(\beta=-0.0026,95 \% \mathrm{Cl}=-0.0041--0.0011, P=0.001)$, fibrin degradation products (FDP; $\beta=0.154,95 \% \mathrm{Cl}=0.023-0.285, P=0.021)$, D-dimer $(\beta=0.0821,95 \% \mathrm{Cl}=$ 0.0135-0.1507, $P=0.019$ ), antithrombin III (AT III; $\beta=-0.0015,95 \% \mathrm{Cl}=-0.1307--0.0107, P=0.021$ ) and total volume of infusion per day (coefficient $=-17.8331,95 \% \mathrm{Cl}=-25.2211--10.445, P<0.001$ ). Although not statistically significant, serum SDC-1 levels from the day before also tended to affect to LD ( $\beta=$ $0.0012,95 \% \mathrm{Cl}=0-0.0024, P=0.056)$. Moreover, the interaction term of time and SDC-1 was significant for AST $(P<0.001)$, ALT $(P<0.001)$, LD $(P<0.001)$, ALP $(P<0.001)$, CRE $(P=0.009)$, BUN $(P=0.018)$, T-Bil $(P=0.001)$ and total volume of infusion per day $(P<0.001)$.

Predicted parameter values over time by serum SDC-1 level 
Figure 1 depicts the linear predicted value of each parameter over time in patients with serum SDC-1 levels of $16.67 \mathrm{ng} / \mathrm{mL}, 45.78 \mathrm{ng} / \mathrm{mL}$ and $318.11 \mathrm{ng} / \mathrm{mL}$, which represent the 25th, 50th and 75th percentile of serum SDC-1, respectively, derived from the mixed effects models summarized in Table 2.

AST, ALT, LD, CRE, BUN, FDP and D-dimer levels were higher in patients with serum SDC-1 levels of $318.11 \mathrm{ng} / \mathrm{mL}$ (high serum SDC-1 levels) than in patients with serum SDC-1 levels of $16.67 \mathrm{ng} / \mathrm{mL}$ and $45.78 \mathrm{ng} / \mathrm{mL}$ (low serum SDC-1 levels). Total volume of infusion per day, T-Bil and AT III levels were lower in patients with high serum SDC-1 levels than in patients with low serum SDC-1 levels.

Differences in AST, ALT, LD, CRE and BUN levels among patients with high and low serum SDC-1 levels increased over time. In contrast, differences in FDP and AT III levels among these patients changed over time but converged to the same value at the end of observational period. Moreover, differences in D-dimer and T-Bill levels and total volume of infusion per day among these patients reversed over time.

\section{Relationship between serum SDC-1 levels and mortality in critically ill patients}

Time-varying Cox proportional hazards regression showed that increasing serum SDC-1 level was significantly correlated with mortality after adjustment for age (hazard ratio for an increase in SDC-1 from 24.23 to $143.91=2.08,95 \% \mathrm{Cl}=1.24-3.49, P=0.006$; Table 3 ).

\section{Discussion}

Serum SDC-1 levels measured the day before laboratory parameter measurements had significant main effects on several outcomes including AST, ALT, T-Bil, CRE, BUN, FDP, D-dimer, AT III and total volume of infusion per day, and showed a tendency to have an effect on LD. Additionally, serum SDC-1 levels from the day before significantly modified the effect between time and several outcomes, including AST, ALT, LD, CRE, BUN, T-Bil and total volume of infusion per day. Moreover, increasing serum SDC-1 level was a significant risk factor for mortality.

AST, ALT, LD, CRE and BUN levels were higher in patients with serum SDC-1 levels from the day before of $318.11 \mathrm{ng} / \mathrm{mL}$ than in those with levels of $16.67 \mathrm{ng} / \mathrm{mL}$ and $45.78 \mathrm{ng} / \mathrm{mL}$, with differences increasing with time from ICU admission. Oda et al. performed a retrospective observational study showing that AST and BUN were closely related to serum SDC-1 in 1313 patients who underwent comprehensive medical examinations. ${ }^{19}$ Similarly, Johansson et al. showed that serum SDC-1 was correlated with CRE $(r=0.34$, $P=0.012)$ in septic patients who received noradrenaline. ${ }^{23}$ Moreover, Puskarich et al. reported that among patients with severe sepsis or septic shock, serum SDC-1 levels were significantly higher in patients with acute kidney injury (AKI) than in those without. ${ }^{21}$ These results are consistent with our present results. Given that AST and ALT are elevated in liver injury, and CRE and BUN are increased in renal injury, serum SDC-1 may be a useful biomarker for daily monitoring of liver and kidney injury in critically ill patients. Moreover, LD is a cytoplasmic enzyme that is widely expressed in tissues such as the 
heart, lungs, kidneys liver, and brain, and is released by these tissues when they are damaged. ${ }^{26,27}$ Further studies are needed to elucidate the relationship between SDC-1 level and heart, lung and brain injuries in critically ill patients.

In contrast, T-Bil and total volume of infusion per day were lower in patients with serum SDC-1 levels from the day before of $318.11 \mathrm{ng} / \mathrm{mL}$ than in those with levels of $16.67 \mathrm{ng} / \mathrm{mL}$ and $45.78 \mathrm{ng} / \mathrm{mL}$. These differences observed from ICU admission reversed with time. Johansson et al. reported that positivity for serum SDC-1 correlated with bilirubin levels $(r=0.45, P=0.001)$ in septic patients who received noradrenaline (23). However, in the present study, T-Bil levels were within the normal range in almost all patients at ICU admission, and any changes in T-Bil within the normal range may not be reflected in the serum SDC-1 level.

Total volume of infusion per day from ICU admission to about 10 days after was lower in patients with serum SDC-1 levels from the day before of $318.11 \mathrm{ng} / \mathrm{mL}$ than in those with levels of $16.67 \mathrm{ng} / \mathrm{mL}$ and $45.78 \mathrm{ng} / \mathrm{mL}$. Inkinen et al. demonstrated that serum SDC-1 levels in 619 adult patients admitted to the ICU did not differ among fluid administration tertiles during the first day in ICU [1 st tertile (less than $201 \mathrm{~mL} / \mathrm{h}$ fluid): $105.7 \mathrm{~mL} / \mathrm{h}$ (56.65-205.20), 2nd tertile (202-314 mL/h): $109.05 \mathrm{~mL} / \mathrm{h}$ (64.93-194.25), 3rd tertile (315-2824 mL/h): $114.45 \mathrm{~mL} / \mathrm{h}(64.08-231.85), P=0.276] .{ }^{28}$ Similarly, Puskarich et al. reported that the administered crystalloid volume in patients with severe sepsis did not differ between high and low SDC-1 groups [4.0 L (IQR 3.3-5.3) vs 3.5. L (IQR 2.4-5.0); $P=0.36] .{ }^{29}$ These results are mostly consistent with the present data. However, serum SDC-1 significantly modified the effect between time and total volume of infusion per day. Differences in total volume of infusion per day between patients with serum SDC-1 levels from the day before of $318.11 \mathrm{ng} / \mathrm{mL}$ and those with levels of $16.67 \mathrm{ng} / \mathrm{mL}$ and $45.78 \mathrm{ng} / \mathrm{mL}$ reversed with time. Thus, further studies are needed to elucidate the relationship between volume of infusion and SDC-1 level.

While serum SDC-1 had significant main effects on several outcomes including AT III, FDP and D-dimer, it did not modify the effect between time and these outcomes. AT III levels were lower in patients with serum SDC-1 levels from the day before of $318.11 \mathrm{ng} / \mathrm{mL}$ than in those with levels of $16.67 \mathrm{ng} / \mathrm{mL}$ and $45.78 \mathrm{ng} / \mathrm{mL}$. AT III levels decrease in critically ill patients due to loss of AT III from the circulation into tissues through increased blood vessel permeability induced by endothelial damage, and decreased production of AT III in the liver and inactivation of AT III by the enzyme elastase. ${ }^{30,31}$ Thus, considering that serum SDC-1 is the core protein of heparan sulfate proteoglycan, a constituent of endothelial glycocalyx, it makes sense that serum SDC-1 had significant main effects on AT III.

FDP and D-dimer are the most important markers for diagnosis of disseminated intravascular coagulation (DIC), which is induced by endothelial injury. ${ }^{32}$ In a prospective observational study of patients with sepsis, lkeda et al reported that there was a weak negative correlation between serum SDC-1 levels and AT III $(r=-0.29, P=0.073) .{ }^{33}$ Moreover, serum SDC- 1 levels on Day 1 were significantly higher in patients with than without DIC [221.4 (189.1-1301.0) vs 107 (60.0-194.5), $P<0.001]$ and had strong discriminative power for predicting both DIC development and mortality (development of DIC: Area under 
the curve $(A \cup C)=0.790 ; 28$-day mortality: $A U C=0.850) .{ }^{33}$ These results are mostly consistent with the present data, suggesting that serum SDC-1 may be a useful biomarker for daily monitoring of the development of DIC.

Differences in AT III and FDP between patients with serum SDC-1 levels from the day before of $318.11 \mathrm{ng} / \mathrm{mL}$ and those with levels of the $16.67 \mathrm{ng} / \mathrm{mL}$ and $45.78 \mathrm{ng} / \mathrm{mL}$ changed over time but converged to the same value at the end of observational period. Further, differences in D-dimer between patients with serum SDC-1 levels from the day before of $318.11 \mathrm{ng} / \mathrm{mL}$ and those with levels of $16.67 \mathrm{ng} / \mathrm{mL}$ and $45.78 \mathrm{ng} / \mathrm{mL}$ reversed with time. Although the mechanisms underlying these changes are unclear, these parameters may have been affected by medical interventions such as AT III replacement therapy and treatment for DIC.

Taken together, our data suggest that serum SDC-1 may be a useful biomarker for daily monitoring of critically ill patients with kidney, liver and coagulation system injuries. Systemic inflammatory conditions such as sepsis induce multiple organ dysfunctions in critically ill patients. Given the wide distribution of endothelial glycocalyx throughout the body, serum SDC-1 may also be a useful biomarker for daily monitoring of injuries to other organs such as the heart, lungs and brain.

There were several limitations in the present study. In addition to endothelial glycocalyx, SDC-1 is also expressed in other organs. We did not evaluate SDC-1 expression in other organs or its associated effects. Additionally, we were unable to evaluate the extent of endothelium injury and serum cytokine levels (e.g., IL-6, TNF-a, NGAL). Finally, the sample size was small and data were obtained from a single institution.

In conclusion, serum SDC-1 may be a useful biomarker for daily monitoring of potential early signs of kidney, liver and coagulation system injury, in addition to endothelium injury. Elevated serum SDC-1 levels may be an important risk factor for mortality in critically ill patients.

\section{Methods}

\section{Patients}

This single-center retrospective observational study was conducted at Gifu University Hospital, which is affiliated with Gifu University (Gifu, Japan). Patients admitted to the intensive care unit (ICU) at Gifu University Hospital from March 2019 to February 2020 were enrolled in the present study. Patients who were younger than 18 years old, were undergoing hemodiafiltration, and were discharged from the ICU within 72 hours were excluded from the analysis.

\section{Ethics approval and consent to participate}

The investigation conformed with the principles outlined in the Declaration of Helsinki. ${ }^{34}$ Ethical approval was obtained from the medical ethics committee of Gifu University Graduate School of Medicine, Gifu, 
Japan (Institutional review board approval No. 2018 - 167). All patients provided written informed consent before all study-related procedures. Before initiation, the study was registered to the UMIN Clinical Trials Registry (registry number: UMIN000036261).

Data collection and study design

On admission to the ICU, blood was routinely sampled every morning from eligible patients, and data from blood samples obtained while patients were in the ICU were used in the present analysis. All laboratory data except serum SDC-1, volume of infusion of maintenance fluid and extracellular fluid, and other patient demographics were extracted from the hospital's electronic medical records. Serum SDC-1 concentrations were measured using an enzyme-linked immunosorbent assay (950.640.192; Diaclone, Besancon, Cedex, France). These data were retrospectively analyzed.

\section{Statistical analysis}

Patients' baseline characteristics are presented as median and interquartile range (IQR) for continuous variables, and frequency and proportion for categorical variables. The aim of study was to identify organ dysfunction that may be amenable to early detection through daily monitoring of SDC-1 levels. To evaluate the effect of SDC-1 on laboratory parameters measured the day after SDC-1 measurement with consideration for repeated measures, we constructed linear mixed effect models, with each parameter as an outcome variable. The models were adjusted for age and sex, and included the interaction term between measurement time and SDC-1. If the interaction term was statistically significant, SDC-1 was determined to have a modifying effect. Additionally, we confirmed the effect of SDC-1 using the main effect of SDC-1, regardless of whether or not the interaction was statistically significant. To normalize residual values, creatine kinase (CK), aspartate aminotransferase (AST), alanine aminotransferase (ALT), lactate dehydrogenase (LD), alkaline phosphatase (ALP), cholinesterase (ChE), creatinine (CRE), blood urea nitrogen (BUN), triglycerides (TG) and T-bilirubin (T-Bil) were natural logarithmically transformed. A time-dependent Cox proportional hazards regression model with adjustment for age was used to evaluate the effect of serum SDC-1 level on mortality. Serum SDC-1 levels were treated as a time-varying variable. A two-sided significance level of $5 \%$ was used to indicate statistical significance. $R$ version 3.6.2 was used for all analyses (www.r-project.org).

\section{Declarations}

Funding: This work was supported by JSPS KAKENHI Grant Number 20K17856, 19H03756, 19K09410, 19K18347, 18K08914, 18K08884, 18K16511, 17K11569 and 20K07587.

\section{Acknowledgments:}

We would like to thank DMC Corp. (www.dmed.co.jp <http://www.dmed.co.jp/>) for English language editing of this paper. 


\section{Author Contributions:}

Keiko S., H.O. and A.S. wrote the manuscript. Yoshinori K., Yuichiro K., Kodai S., N.Y., T.M., T.F., R.Y., H.O., T.D. and T.Y. collected blood samples. Keiko S., Kazuyuki S. and R.K. measured SDC-1 using ELISA. Keiko S. and Kazuyuki S. created the database, T.I. performed statistical analysis. H.T. and S.Y. supervised the study. H.O., S.O. and A.S. revised and edited the manuscript. All authors read and approved the final manuscript.

Competing Interests: The authors declare no competing interests.

\section{References}

1 Luft, J. H. Fine structures of capillary and endocapillary layer as revealed by ruthenium red. Federation proceedings25, 1773-1783 (1966).

2 Salmon, A. H. \& Satchell, S. C. Endothelial glycocalyx dysfunction in disease: albuminuria and increased microvascular permeability. The Journal of pathology226, 562-574, doi:10.1002/path.3964 (2012).

3 Paulus, P., Jennewein, C. \& Zacharowski, K. Biomarkers of endothelial dysfunction: can they help us deciphering systemic inflammation and sepsis? Biomarkers : biochemical indicators of exposure, response, and susceptibility to chemicals16 Suppl 1, S11-21, doi:10.3109/1354750x.2011.587893 (2011).

4 Li, L., Ly, M. \& Linhardt, R. J. Proteoglycan sequence. Molecular bioSystems8, 1613-1625, doi:10.1039/c2mb25021g (2012).

5 Woodcock, T. E. \& Woodcock, T. M. Revised Starling equation and the glycocalyx model of transvascular fluid exchange: an improved paradigm for prescribing intravenous fluid therapy. British journal of anaesthesia108, 384-394, doi:10.1093/bja/aer515 (2012).

6 Frati-Munari, A. C. [Medical significance of endothelial glycocalyx]. Archivos de cardiologia de Mexico83, 303-312, doi:10.1016/j.acmx.2013.04.015 (2013).

7 Lee, W. L. \& Slutsky, A. S. Sepsis and endothelial permeability. The New England journal of medicine363, 689 (2010).

8 Reitsma, S., Slaaf, D. W., Vink, H., van Zandvoort, M. A. \& oude Egbrink, M. G. The endothelial glycocalyx: composition, functions, and visualization. Pflugers Archiv : European journal of physiology454, 345-359, doi:10.1007/s00424-007-0212-8 (2007).

9 Schmidt, E. P. et al. The pulmonary endothelial glycocalyx regulates neutrophil adhesion and lung injury during experimental sepsis. Nature medicine18, 1217-1223, doi:10.1038/nm.2843 (2012). 
10 Schmidt, E. P. et al. The circulating glycosaminoglycan signature of respiratory failure in critically ill adults. The Journal of biological chemistry289, 8194-8202, doi:10.1074/jbc.M113.539452 (2014).

$11 \mathrm{Okada}, \mathrm{H}$. et al. Three-dimensional ultrastructure of capillary endothelial glycocalyx under normal and experimental endotoxemic conditions. Critical care (London, England)21, 261, doi:10.1186/s13054-0171841-8 (2017).

12 Inagawa, R. et al. Ultrastructural Alteration of Pulmonary Capillary Endothelial Glycocalyx During Endotoxemia. Chest154, 317-325, doi:10.1016/j.chest.2018.03.003 (2018).

13 Ando, Y. et al. Brain-Specific Ultrastructure of Capillary Endothelial Glycocalyx and Its Possible Contribution for Blood Brain Barrier. Scientific reports8, 17523, doi:10.1038/s41598-018-35976-2 (2018).

14 Uchimido, R., Schmidt, E. P. \& Shapiro, N. I. The glycocalyx: a novel diagnostic and therapeutic target in sepsis. Critical Care23, 16 (2019).

15 Padberg, J.-S. et al. Damage of the endothelial glycocalyx in chronic kidney disease. Atherosclerosis234, 335-343 (2014).

16 Wang, J.-b. et al. Insulin increases shedding of syndecan-1 in the serum of patients with type 2 diabetes mellitus. Diabetes research and clinical practice86, 83-88 (2009).

17 Broekhuizen, L. N. et al. Effect of sulodexide on endothelial glycocalyx and vascular permeability in patients with type 2 diabetes mellitus. Diabetologia53, 2646-2655 (2010).

18 Neves, F. M. et al. Syndecan-1 in Acute Decompensated Heart Failure--Association With Renal Function and Mortality. Circulation journal : official journal of the Japanese Circulation Society79, 1511-1519, doi:10.1253/circj.CJ-14-1195 (2015).

19 Oda, K. et al. Factors Enhancing Serum Syndecan-1 Concentrations: A Large-Scale Comprehensive Medical Examination. Journal of clinical medicine8, doi:10.3390/jcm8091320 (2019).

20 Ostrowski, S. R. et al. Association between biomarkers of endothelial injury and hypocoagulability in patients with severe sepsis: a prospective study. Critical Care19, 191 (2015).

21 Puskarich, M. A., Cornelius, D. C., Tharp, J., Nandi, U. \& Jones, A. E. Plasma syndecan-1 levels identify a cohort of patients with severe sepsis at high risk for intubation after large-volume intravenous fluid resuscitation. Journal of critical care36, 125-129 (2016).

22 Sallisalmi, M., Tenhunen, J., Yang, R., Oksala, N. \& Pettilä, V. Vascular adhesion protein-1 and syndecan-1 in septic shock. Acta Anaesthesiologica Scandinavica56, 316-322 (2012).

23 Johansson, P. I., Haase, N., Perner, A. \& Ostrowski, S. R. Association between sympathoadrenal activation, fibrinolysis, and endothelial damage in septic patients: a prospective study. Journal of critical 
care29, 327-333 (2014).

24 Anand, D., Ray, S., Srivastava, L. M. \& Bhargava, S. Evolution of serum hyaluronan and syndecan levels in prognosis of sepsis patients. Clinical biochemistry49, 768-776 (2016).

25 Ostrowski, S. R., Gaïni, S., Pedersen, C. \& Johansson, P. I. Sympathoadrenal activation and endothelial damage in patients with varying degrees of acute infectious disease: an observational study. Journal of critical care30, 90-96 (2015).

26 Ballas, S. K. Lactate dehydrogenase and hemolysis in sickle cell disease. Blood121, 243-244 (2013).

27 Riske, L., Thomas, R. K., Baker, G. B. \& Dursun, S. M. Lactate in the brain: an update on its relevance to brain energy, neurons, glia and panic disorder. Therapeutic advances in psychopharmacology7, 85-89 (2017).

28 Inkinen, N. et al. Association of endothelial and glycocalyx injury biomarkers with fluid administration, development of acute kidney injury, and 90-day mortality: data from the FINNAKI observational study. Annals of intensive care9, 103 (2019).

29 Puskarich, M., Cornelius, D., Tharp, J., Nandi, U. \& Jones, A. 385 Plasma Syndecan-1 Levels Identify a Cohort of Patients With Severe Sepsis at a High Risk of Intubation Following Large Volume Intravenous Fluid Resuscitation. Annals of Emergency Medicine4, S147 (2016).

30 Jordan, R. E., Kilpatrick, J. \& Nelson, R. M. Heparin promotes the inactivation of antithrombin by neutrophil elastase. Science237, 777-779 (1987).

31 Seitz, R., Wolf, M., Egbring, R. \& Havemann, K. The disturbance of hemostasis in septic shock: role of neutrophil elastase and thrombin, effects of antithrombin III and plasma substitution. European journal of haematology43, 22-28 (1989).

32 Asakura, H. et al. Proposal for new diagnostic criteria for DIC from the Japanese Society on Thrombosis and Hemostasis. Thrombosis Journa/14, 42 (2016).

33 Ikeda, M. et al. Circulating syndecan-1 predicts the development of disseminated intravascular coagulation in patients with sepsis. Journal of Critical Care43, 48-53 (2018).

34 Rickham, P. P. Human Experimentation. Code of Ethics of the World Medical Association. Declaration of Helsinki. Br Med J2, 177, doi:10.1136/bmj.2.5402.177 (1964).

\section{Tables}




\begin{tabular}{|ll|}
\hline Table 1. Patient demographics & \\
\hline Age, years, median (IQR) & $67(53.3-77.0)$ \\
\hline Sex, male/female, $\mathrm{n}(\%)$ & $70(74.5) / 24(25.5)$ \\
\hline Weight, kg, median (IQR) & $60.4(53.1-75.1)$ \\
\hline SOFA score on ICU admission, median (IQR) & $6.0(4.0-9.8)$ \\
\hline Condition, $\mathrm{n}(\%)$ & \\
\hline Trauma & $32(34.0)$ \\
\hline Myocardial infarction & $15(16.0)$ \\
\hline Soft tissue infection & $7(7.4)$ \\
\hline Burn & $6(6.4)$ \\
\hline Heatstroke & $5(5.3)$ \\
\hline Heart failure & $3(3.2)$ \\
\hline Loss of consciousness & $2(2.1)$ \\
\hline Acute aortic dissection & $2(2.1)$ \\
\hline Ventricular fibrillation & $2(2.1)$ \\
\hline Other & $17(18.1)$ \\
\hline Length of ICU stay, days, median (IQR) & $10.5(6.0-19.0)$ \\
\hline All data indicate median, 25-75th percentile unless otherwise indicated \\
\hline
\end{tabular}

ICU: intensive care unit, IQR: interquartile range 
Table 2. Linear mixed effects models that did and did not consider the interaction of time and SDC-1

No consideration for interaction of time and SDC-1

Outcome Coefficient* $95 \% \mathrm{LCL} \quad 95 \% \mathrm{UCL} \quad P$-value $P$-value for interaction of time and SDC-1

$\begin{array}{llllll}\text { TP } & 0.0012 & -0.0037 & 0.0061 & 0.638 & 0.362 \\ \text { ALB } & 0.0015 & -0.0013 & 0.0042 & 0.289 & 0.111\end{array}$

$\begin{array}{llllll}\text { log_CK } & 0.0013 & -0.0017 & 0.0043 & 0.401 & 0.861\end{array}$

$\begin{array}{llllll}\text { log_AST } & 0.0042 & 0.0018 & 0.0065 & 0.001 & <.001\end{array}$

$\begin{array}{llllll}\text { log_ALT } & 0.0034 & 0.0012 & 0.0057 & 0.003 & <.001\end{array}$

$\begin{array}{llllll}\text { log_LD } & 0.0012 & 0 & 0.0024 & 0.056<0.001\end{array}$

$\begin{array}{llllll}\text { log_ALP } & 0.0002 & -0.0010 & 0.0015 & 0.695 & 0.001\end{array}$

$\begin{array}{llllll}\text { log_ChE } & -0.0014 & -0.0032 & 0.0004 & 0.115 & 0.365\end{array}$

$\begin{array}{llllll}\text { log_CRE } & 0.0020 & 0.0012 & 0.0028 & <.001 & 0.009\end{array}$

$\begin{array}{llllll}\text { log_BUN } & 0.0015 & 0.0002 & 0.0028 & 0.019 & 0.018\end{array}$

$\begin{array}{llllll}\text { log_TG } \quad 0.0032 & -0.0455 & 0.0519 & 0.874 & 0.805\end{array}$

$\begin{array}{llllll}\text { log_T-Bil } & -0.0026 & -0.0041 & -0.0011 & 0.001 & 0.004\end{array}$

$\begin{array}{llllll}\text { FDP } & 0.1540 & 0.0230 & 0.2850 & 0.021 & 0.336\end{array}$

$\begin{array}{llllll}\text { D-dimer } & 0.0821 & 0.0135 & 0.1507 & 0.019 & 0.152\end{array}$

$\begin{array}{llllll}\text { AT III } & -0.0707 & -0.1307 & -0.0107 & 0.021 & 0.694\end{array}$

$\begin{array}{lllll}\text { Volume of infusion } & -17.8331 & 25.2211 & -10.4450<0.001<0.001\end{array}$ per day

*Coefficient indicates the increase in the mean value of an outcome when SDC-1 increases by 10. SDC-1: syndecan-1; TP: total protein; ALB: albumin; CK: creatine kinase; AST: aspartate aminotransferase; ALT: alanine aminotransferase; LD: lactate dehydrogenase, ALP: alkaline phosphatase; ChE: cholinesterase; CRE: creatinine; BUN: blood urea nitrogen; TG: triglyceride; T-Bil: total-bilirubin; FDP: fibrin degradation product; AT III: antithrombin III, LCL: lower confidence limit, UCL: upper confidence limit

All mixed effects models were adjusted for age and sex. 
Table 3. Time-dependent Cox proportional hazards regression analysis of mortality in critically ill patients

$\begin{array}{lllll}\text { Factor } & \text { HR } & 95 \% \mathrm{LCl} & 95 \% \mathrm{UCl} & P \text {-value } \\ \text { Serum SDC-1 level (IQR: 24.2-143.9) } & 2.08 & 1.24 & 3.49 & 0.006 \\ \text { Age (IQR: } 54-78.5) & 1.93 & 0.53 & 6.95 & 0.317\end{array}$

HR: hazard ratio for an increase in each factor from the 25 percentile to the 75 percentile, IQR: interquartile range, LCL: lower confidence limit, UCL: upper confidence limit, SDC-1: syndecan-1

\section{Figures}
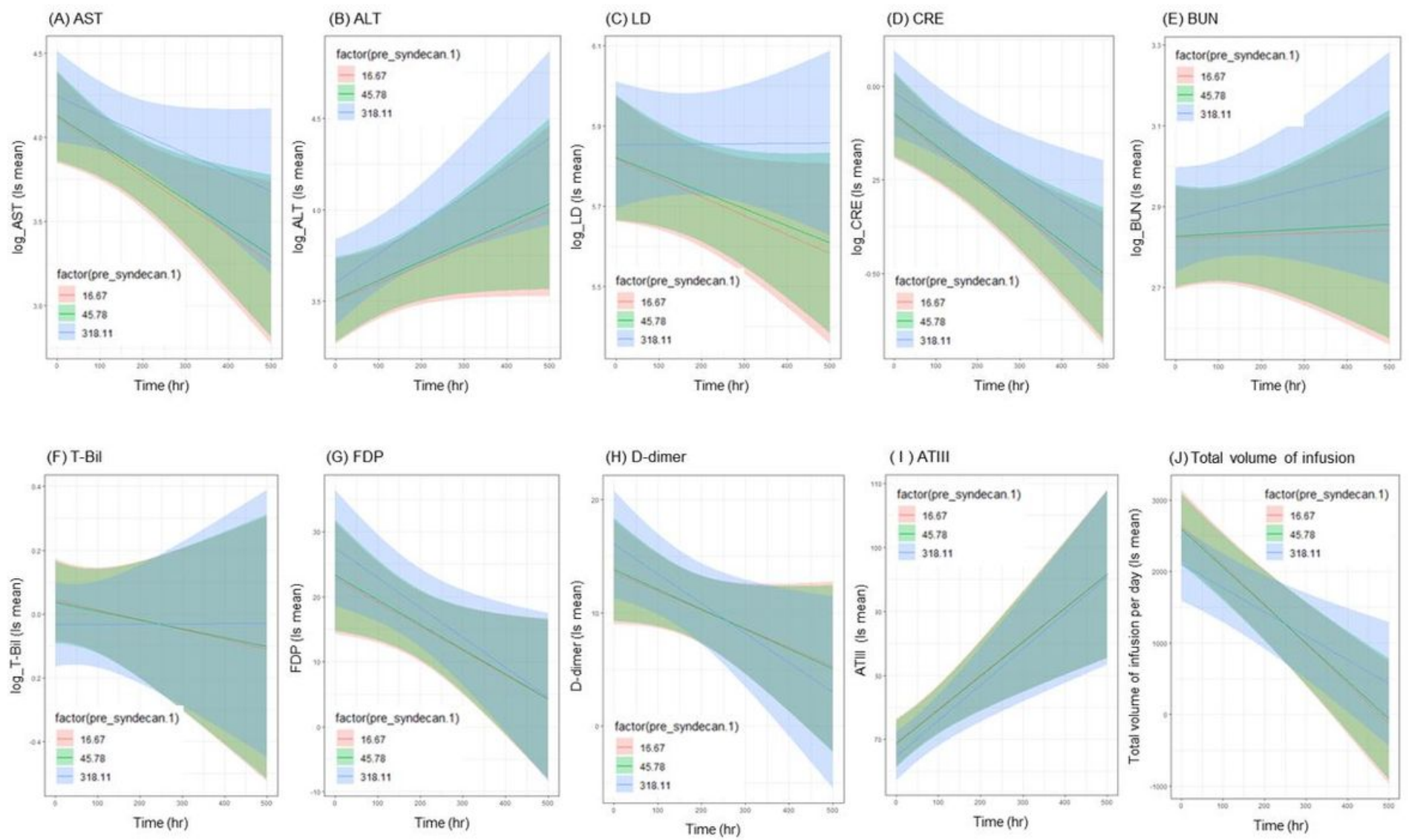

\section{Figure 1}

Predicted value of each parameter over time in patients with serum SDC-1 levels from the day before of $16.67 \mathrm{ng} / \mathrm{mL}$ (red line), $45.78 \mathrm{ng} / \mathrm{mL}$ (green line), and $318.11 \mathrm{ng} / \mathrm{mL}$ (blue line). 